\title{
Simulación del proceso de compactación de polvos metálicos
}

\author{
J.M. Prado ${ }^{(*)}$ y M.D. Riera ${ }^{(*)}$
}

\begin{abstract}
Resumen La simulación mediante elementos finitos de procesos de conformación de componentes mecánicos es una herramienta cada vez más utilizada para su diseño y validación. En este trabajo se presenta la simulación de la compactación en molde de polvo metálico para la elaboración de una pieza pulvimetalúrgica. Se aplica el modelo de plasticidad CAM-clay modificado y se resuelve con el software de cálculo Abaqus. El resultado, en forma de distribuciones de densidades, permite determinar el movimiento de punzones que optimiza la calidad de la pieza acabada. Así mismo, se obtiene la distribución de tensiones en las distintas zonas del molde.
\end{abstract}

Palabras clave: Pulvimetalurgia. Polvos metálicos. Compactación. Simulación de procesos.

\section{Simulation of the metallic powders compaction process}

\begin{abstract}
The simulation by means of finite elements of the forming processes of mechanical components is a very useful tool for their design and validation. In this work, the simulation of the compaction of a metal powder is presented. The finite element software Abaqus is used together with the modified CAM-clay plasticity model in order to represent the elastoplastic behaviour of the material. Density distributions are obtained and, therefore, the motion of the compaction punches which improve this distribution can be found. Stress distribution in the different parts of the mould can also be determined.
\end{abstract}

Keywords: Powder metallurgy. Metallic powders. Compaction. Processes simulation.

\section{INTRODUCCIÓN}

La compactación del polvo metálico dentro del molde, que le confiere la forma deseada, es una etapa fundamental en la elaboración de piezas pulvimetalúrgicas. En esta fase queda determinada la distribución de densidades en la pieza, ya que la sinterización posterior confiere al compacto la resistencia mecánica necesaria, pero no puede eliminar los defectos introducidos durante la compactación. La distribución de densidades puede controlarse a través de la geometría de la pieza y, sobre todo, mediante el diseño adecuado del proceso de compactación: adaptando cuidadosamente los movimientos relativos de los punzones que comprimen el polvo metálico en el interior del molde.

La definición detallada del proceso de compactación puede optimizarse mediante "simulación". A

(*) Dpto. de Ciencia de Materiales e Ingeniería Metalúrgica. Univ. Politécnica de Cataluña. E.T.S.E.I.B. Avda. Diagonal, 647. 08028-Barcelona (España). pesar de que sólo un sistema experto lleva al diseño completo, la utilización de un software de cálculo por elementos finitos permite obtener información de gran interés desde el punto de vista del comportamiento del material y la influencia de las herramientas y parámetros del conformado.

El primer aspecto a tener en cuenta es el modelo de comportamiento mecánico del material a compactar que es, actualmente, objeto de un gran esfuerzo investigador (1). El modelo clásico de fluencia de los metales totalmente densos, incompresibles, de von Mises, no puede aplicarse a este caso, ya que, durante la compresión de un material granular, hay densificación y, por lo tanto, la componente hidrostática de la tensión juega un papel fundamental.

De los distintos modelos propuestos (2-4), el más sencillo es el de plasticidad de CAM-clay (o CAM-clay modificado) (5). En este caso, la superficie de cedencia en el espacio "componente hidrostática de la tensión, $p$ - componente desviadora de la tensión, $q$ " consiste en una recta (la recta 
"crítica") que pasa por el origen y que separa la zona de densificación (a la derecha de la recta) de la de desconsolidación, o fallo (zona de la izquierda de dicha recta). Los sucesivos estados de endurecimiento del material durante la compresión, se representan mediante elipses (Fig. 1).

En este trabajo se presentan los resultados obtenidos en la simulación de la compactación en frío del polvo prealeado, de Höganäs, Distalloy-AE, utilizando este modelo de comportamiento, a fin de mostrar su capacidad para predecir el efecto que sobre la distribución de densidades ejercen los distintos parámetros del proceso industrial.

\section{MODELO DE COMPORTAMIENTO DEL MATERIAL}

Las expresiones matemáticas del modelo de plasticidad CAM-clay son las siguientes:

Comportamiento elasto-plástico:

$$
e-e_{\mathrm{o}}=\lambda\left(\ln p_{\mathrm{o}}-\ln p\right)
$$

Locus de cedencia:

$$
M^{2} p-M^{2} p_{o} p+q^{2}=0
$$

donde $e$ es la proporción residual de poros; $\lambda$, el módulo plástico; $p$, la componente hidrostática de la tensión; $q$, la componente desviadora de la tensión, y $M$, la pendiente de la recta crítica.

Para representar el comportamiento elástico, fundamental sobre todo en la etapa de eyección del compacto, se ha utilizado un modelo típicamente aplicado a materiales geológicos (6-8) y que relaciona exponencialmente la variación de la proporción residual de poros elástica con la componente hidrostática de la tensión aplicada, tal como se muestra en la siguiente ecuación:

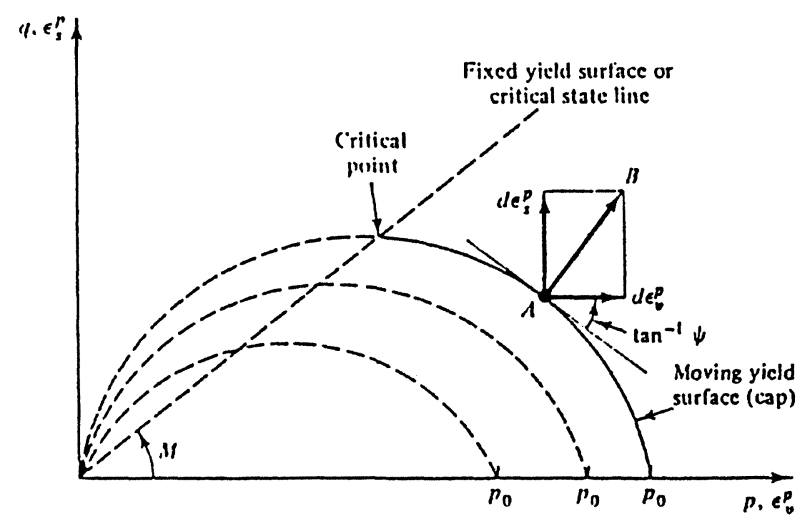

FIG. 1.-Locus de cedencia en el espacio $p-q$.

FIG. 1.- Locus of yield in the space $\mathrm{p}-\mathrm{q}$.

$$
e^{\mathrm{el}}-e_{\mathrm{o}}^{\mathrm{el}}=k\left(\ln p_{\mathrm{o}}-\ln p\right)
$$

en la que $k$ es el módulo volumétrico elástico.

El significado de cada uno de los parámetros de los modelos expuestos se muestra en las figuras 1 y 2 .

\section{SIMULACIÓN}

Se ha simulado la fabricación del compacto de la figura 3, que corresponde a una geometría típica de la industria pulvimetalúrgica, con el programa de cálculo Abaqus (9). Los valores de los parámetros para los modelos elástico y elastoplástico, se indican en la tabla I.

El molde para la compactación, que se muestra en la figura 4, se ha adaptado a una prensa de efecto múltiple con un punzón superior, uno central y dos inferiores. Se han estudiado dos casos distintos:

Caso 1: Punzones superior e inferior-externo móviles. Punzón central e inferior-interno fijos.

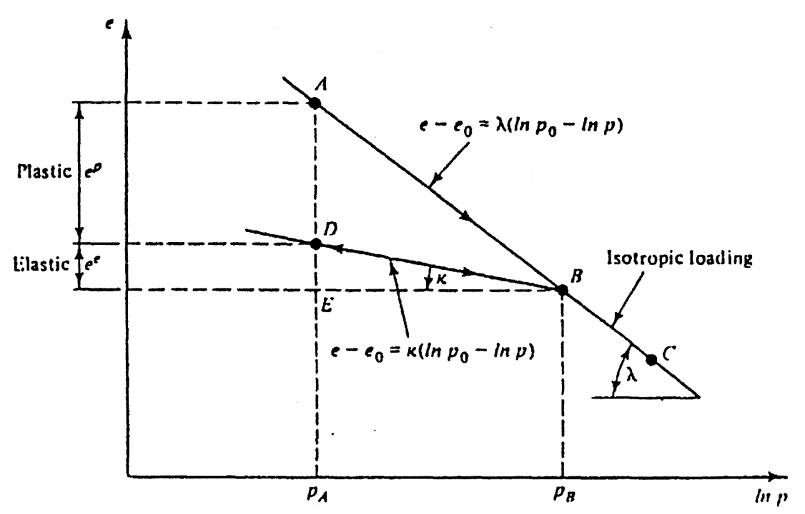

FIG. 2.- Comportamiento a consolidación en el espacio $\ln p-e$.

FIG. 2.- Consolidation behaviour in the space $\ln \mathrm{p}-\mathrm{e}$.

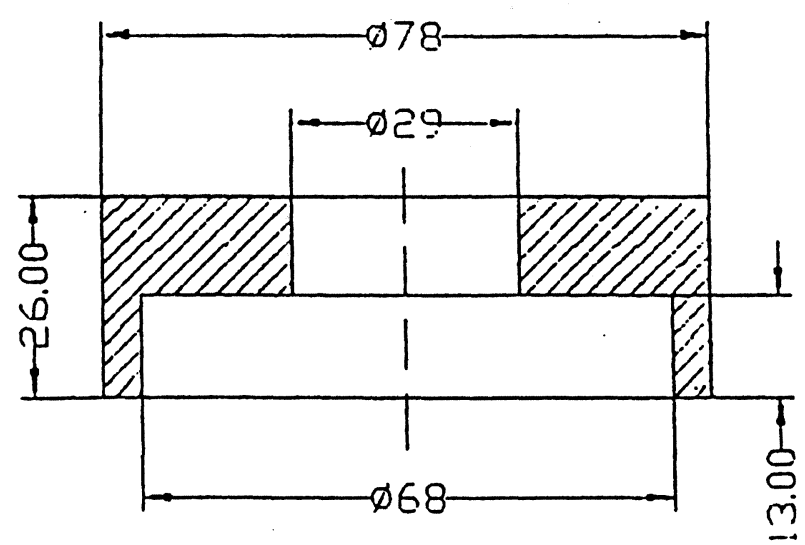

FIG. 3.- Pieza a modelizar.

FIG. 3.-Part to be modelled. 
TABLA I.- Parámetros del comportamiento elasto-plástico del material TABLE I.- Parameters of the elastic-plastic behaviour of the material

\begin{tabular}{|c|c|c|c|c|c|c|c|}
\hline$k$ & $\mathrm{v}$ & $\mathrm{P}_{\mathrm{t}}^{\mathrm{el}} \mathrm{MPa}$ & $\lambda$ & $M$ & $\mathrm{a}_{\mathrm{o}}, \mathrm{MPa}$ & $\beta$ & $K$ \\
\hline 0,005 & 0,3 & 0 & 0,226 & 1,47 & 0,95 & 1 & 1 \\
\hline
\end{tabular}

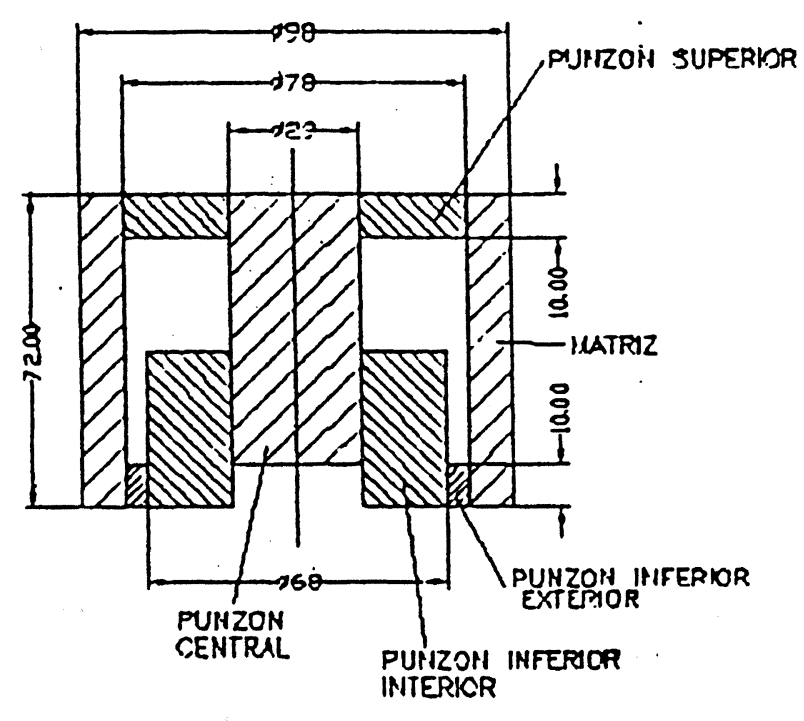

FIG. 4.- Molde y punzones para la compactación.

FIG. 4.- Mould and punches for compaction.

Caso 2: Punzones superior, inferiores-externo e interno móviles. Punzón central fijo.

En ambas situaciones, los movimientos de los punzones son los adecuados para conseguir una densidad teórica uniforme de $6,6 \mathrm{Mg} / \mathrm{m}^{3}$.

\section{RESULTADOS Y DISCUSIÓN}

En la figura 5 se muestra la distribución de la proporción residual de poros, $e$, en la sección de la pieza para el caso 1 . Se puede apreciar en ella que las zonas más densas están localizadas en la región de las esquinas (excepto en la de la zona inferior izquierda, junto al punzón central) y la de menor densidad está localizada en la región de cambio de sección. Aquí se prevé, también, la mayor concentración de esfuerzos de la pieza en su aplicación, con lo que aumenta la posibilidad de fallo en servicio.

En la figura. 6 se presenta la distribución de densidades en el supuesto 2 . Ahora, el recorrido del punzón superior es menor, el punzón inferior-interno es móvil y aumenta su recorrido el punzón inferior-externo. Esta modificación del movimiento relativo de los punzones altera la distribución de densidades respecto del caso anterior, de manera
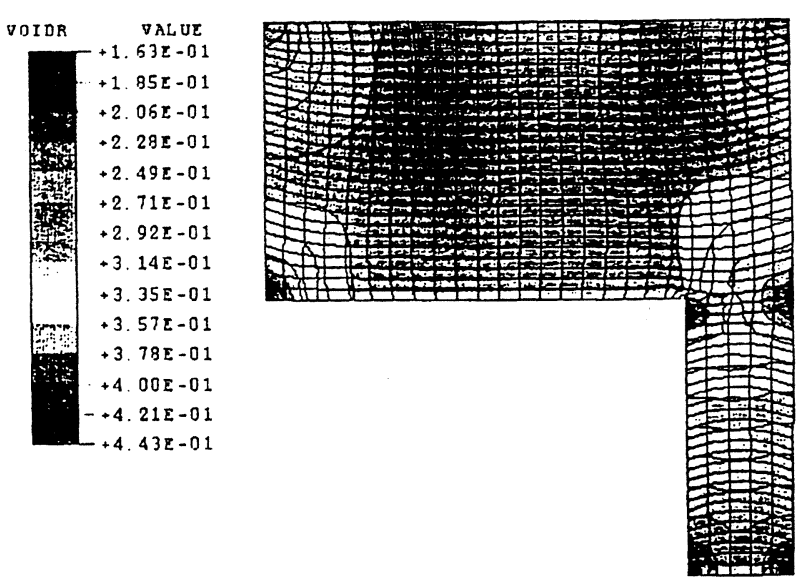

FIG. 5.- Distribución de la proporción de poros en el compacto. Caso 1.

FIG. 5.-Pore distribution in the compact. Case 1.
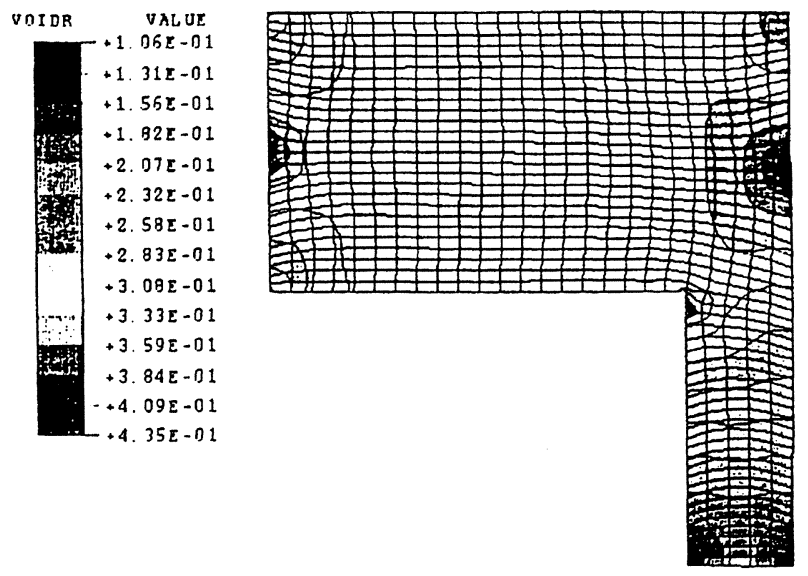

FIG. 6.- Distribución de la proporción de poros en el compacto. Caso 2.

FIG. 6.-Pore distribution in the compact. Case 2.

que, ahora, la densidad mínima no se localiza en la zona crítica de cambio de sección, sino en otra región menos comprometida mecánicamente.

Por último, la figura 7 muestra la distribución de la tensión equivalente en las distintas partes del molde al final de la compactación y para el caso 1 . Se puede apreciar en ella que el nivel de esfuerzos es, en general, relativamente bajo para el tipo de acero rápido utilizado habitualmente en la 

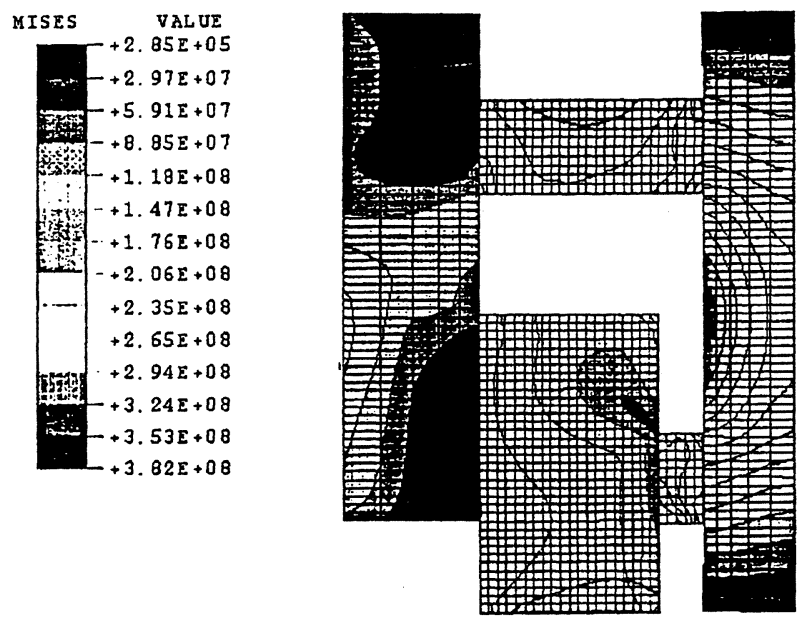

FIG. 7.- Distribución de la tensión equivalente en el molde para el caso 1.

FIG. 7.-Equivalent stress disiribution in the mould for case 1 .

fabricación de los moldes de compactar. La zona más rigurosamente solicitada es la cara interior del cilindro externo del molde; en este caso, podrían aparecer problemas de desgaste si el número de piezas a compactar fuera muy elevado.

\section{CONCLUSIONES}

La simulación mediante elementos finitos del proceso de compactación de polvos metálicos per- mite optimizar el proceso de elaboración de piezas pulvimetalúrgicas.

El modelo de plasticidad CAM-clay modificado representa convenientemente el comportamiento elastoplástico de este tipo de materiales durante su compresión en frío.

\section{REFERENCIAS}

(1) Proc. Intern. Workshop on Modelling of Metal Powder Forming Processes. Grenoble (Francia). 1977.

(2) Gurson, A.L. J. Eng. Met. Tech. Paper No. 76-Mat-CC. 1976.

(3) Shima, S. y Oyane, M. Int. J. Mec. Sci. 18, $1976: 285$.

(4) Fleck, N.A., Kuhn, L.H. y McMeeking, R.M. J. Mech. Phys. Solids. 40 (5), 1992 : 1.139.

(5) Roscoe, K.H., Schofield, A. y Thurairajah, A. Géotechnique. 13 (3), $1963: 211$.

(6) Roscoe, K.H. y Pooroshasb, H.B. Géotechnique. 13 (3), 1963.

(7) ZiENKIEwICZ, O.C. y NAYLOR, D.J. Stress-strain behaviour of Soils. Editorial Parry. 1972: 537.

(8) Riera, M.D. y Prado, J.M. Proc. Intern. Workshop on Modelling of Metal Powder Forming Processes. Grenoble (Francia) $1977: 29$.

(9) H.K.S. Inc. ABAQUS Theory Manual. Versión 5.6. 1996. 